\title{
COORDINATION INDEX FOR DIRECTIONAL OVERCURRENT RELAYS USING MULTIOBJECTIVE C-DEEPSO APPROACH
}

\author{
Wellington Maycon S. Bernardes*, Eduardo Nobuhiro Asada ${ }^{\dagger}$, Jean Sumaili ${ }^{\ddagger}$, Leonel M. \\ Carvalho $\ddagger$, Vladimiro Miranda ${ }^{\S}$ \\ * Federal University of Uberlândia, Faculty of Electrical Engineering \\ Uberlândia, $M G$, Brazil \\ †University of São Paulo, Department of Electrical and Computer Engineering \\ São Carlos, SP, Brazil \\ ${ }^{\ddagger}$ INESC Technology and Science \\ Porto, Portugal \\ $\S$ University of Porto \\ Porto, Portugal
}

Emails: wmsbernardes@ufu.br, easada@usp.br, jean.sumaili@inesctec.pt, lcarvalho@inesctec.pt, vmiranda@inesctec.pt

\begin{abstract}
Directional Overcurrent Relays can be dynamically and quickly configured using optimization techniques making the electrical network more resilient. In this work, the directional overcurrent relay coordination is modeled as mixed integer non-linear problem that minimizes the sum of relays operating times and maximizes their coordination intervals. To solve this, the use of Multiobjective Canonical Differential Evolutionary Particle Swarm Optimization is proposed in this work. The effects of different probabilistic distributions (normal, uniform, triangular and qui-squared ones) to initialize the decision variables (time dials, pickup currents, curves and indexes) are also discussed in this work. With the proposed method a reduction in convergence speed of more than $50 \%$ can be obtained when the adequate probabilistic distribution is used. The decrease in time can also be enhanced if a different acceptable reduction of the Coordination Time Interval is considered resulting in different and robust solutions.
\end{abstract}

Keywords - Power System Protection, Computational intelligence, Optimization, Modelling and Simulation, Blackout Prevention.

\section{Introduction}

The power system is subject to short-circuit currents, also known simply as faults, which may damage certain equipment installed nearby and interrupt temporarily the continuity of the service for the consumers. The fault direction may be forward (from relay ahead) or reverse (Ukil et al., 2011). The normal flow is forward, where the Directional Overcurrent Relay (DOR) operates when the current that flows is greater than and established pickup, similar to a "setpoint". In contrary direction, the protective device remains inoperative. In interconnected system with DORs is important the coordination of each device to avoid misoperation. This consists in following key philosophy: the devices closer the fault should operate firstly, and in case of a failure, its backup device acts after a Coordination Time Interval (CTI).

One simple way to speed up the relay actuation is to reduce the CTI. Electromechanical or microprocessor-based relays have standard range of CTI (generally, between 0.2 and 0.5 sec depending on the type). In the literature normally fixed values are adopted, as $0.3 \mathrm{~s}$, when the calculation is performed (Tjahjono et al., 2017; Elmitwally et al., 2015; Meskin et al., 2015; IEEE, 2001; Noghabi et al., 2010). An adequate CTI depends on several considerations such as: start time of elements (reaction), possible delay of the upstream relay, error margin due to Current Transformer (CT) saturation, digital relays operating time and relay contacts operating time. As these errors are not well known and for safety reasons, greater CTI values are preferred in practice.

The DOR coordination problem (Costa et al., 2017; El-Fergany and Hasanien, 2017) is typically solved by means of mixed integer non-linear programming techniques in order to minimize an objective function, which consists in the sum of DOR operating times (Bottura et al., 2017; Bernardes et al., 2013).

Moreover, the optimal coordination of DORs has been intensively studied in recent years (Rajput et al., 2018; Shabani and Karimi, 2018; Bernardes et al., 2015). The protection systems using DORs are considered as backup protection of protection of transmission systems, working with distance relays, and as primary protection of distribution or sub-transmission. In addition, to help the protection engineers in setting correctly many different protection devices, nowadays the developed tools based on Artificial Intelligence can minimize accidental operation. The main target of this study is to find Time Multiplier Settings (TMSs), taps and curve characteristic of each relay considering constraints as CTI. 
From the above literature, it can be emphasized that a non-fixed CTI may be more useful in practice. Furthermore, there are some difficult situations to be dealt with metaheuristic optimization involving convergence speed and computational time. The improvement of the processing time is directly dependent of the chosen distribution for starting the decision variables. DORs can be dynamically and quickly re-coordinated using optimization techniques after a topology reconfiguration provoked by a disturbance in the system, for example. This make the operation more secure and resilient under adverse conditions.

In this paper, firstly, we propose a more practical approach by using a coordination index (function $f_{2}$ ) to be maximized and not only to minimize the sum of the operating times (function $f_{1}$ ) already very used by many methods in the literature. If the protection specialist wants to find several operating times, knowing that the CTI can be decreased up to a certain admissible level in order to speed up the operation of the devices, this is a plausible reason to map other solutions. In short, verifying different acceptable rates of lower CTI, it is possible to obtain several robust solutions which take into account the balance or trade-off between the CTI and the speed of DOR actuation. Secondly, another improvement is reached when we understand about the potential region that each setting (decision variable) is more favorable to remain after the optimization process. It is significant the correct utilization of probabilistic distribution function in the algorithm to speed up the search. We evaluated how a wrong choice of the distribution function may influence the final results.

Our goal is reached by using a new approach called as Multiobjective Canonical Differential Evolutionary Particle Swarm Optimization (C-DEEPSO) and tested in the IEEE 30-bus system, which involves a large number of decision variables. The multiobjective approach brings an effective set of solutions to the protection engineer. All algorithm is modelled using language $\mathrm{C}++$.

In addition, the results demonstrate that the simulation time decreased more than $50 \%$ when choosing a more accurate probability distribution, triangular or chi-squared distribution, instead of normal or uniform one, being a significant enhancement for on-line application. It is observed that the TMSs as well as the taps or Plug Setting Multipliers (PSMs) tend predominantly toward lowest values. For this reason, these variables are randomly initialized in the optimization process with the majority of the numbers close to the lower bound (decreasing frequency to upper bound).

The remainder of this paper is divided into five sections. The mathematical formulation to coordinate the DORs is discussed in Section 2. Section 3 explains about the Multiobjective C-DEEPSO, a novel technique implemented in this paper. Section 4 deals with the probabilistic distribution for the relay coordination problem. The results are commented in Section 5. Finally, the conclusions are drawn in Section 6.

Our goal is reached by using a new approach called as Multiobjective C-DEEPSO (Marcelino et al., 2016), which derives from the single objective meta-heuristic EPSO (Miranda and Fonseca, 2002). The multiobjective approach brings an effective set of solutions to the protection engineer. The algorithm is implemented using language $\mathrm{C}++$.

\section{Modeling the Operating Time and Coordination Index}

The mathematical modeling that minimizes the sum of operating time of all DORs $\left(f_{1}\right)$ while maximizes the coordination index $\left(f_{2}\right)$ satisfying basic constraints or conditions is described in Equation 1.

$$
\begin{aligned}
& \min . \quad f_{1}=\sum_{i=1}^{N P} \sum_{j=1}^{N R} t_{i j}^{\prime}\left(P S M_{i j}, T M S_{j}, \Phi_{j}\right)+\sum_{i=1}^{N P} \sum_{j=1}^{N R} \sum_{k=1}^{N_{\mathcal{S}_{j}}} \cdots \\
& t_{i j k}^{\prime \prime}\left(P S M_{i j k}, T M S_{j k}, \Phi_{j k}\right) \\
& \max . f_{2}=\frac{\sum_{j=1}^{N R} \sum_{k=1}^{N} \mathcal{S}_{j}}{m} \\
& \text { s.t. } \\
& t_{i j k}^{\prime \prime}-t_{i j}^{\prime} \geq \gamma_{j k} \times C T I_{\max }, \forall i \leq N P, \forall j \leq N R, \forall k \leq N_{\mathcal{S}_{j}} \\
& \sum_{j=1}^{N R} \sum_{k=1}^{N_{\mathcal{S}_{j}}} \gamma_{j k} \geq(1-\alpha) m, \forall j \leq N R, \forall k \leq N_{\mathcal{S}_{j}} \\
& 1-\beta\left(1-e^{-10000 \times \alpha}\right) \leq \gamma_{j k} \leq 1, \forall j \leq N R, \forall k \leq N_{\mathcal{S}_{j}} \\
& t(P S M, T M S, \Phi)=T M S \times \frac{A_{\Phi}}{P S M^{B} \Phi-1}, \forall i \leq N P, \forall j \leq N R, \cdots \\
& \forall k \leq N_{\mathcal{S}_{j}} \\
& P S M=\frac{I_{c c}}{I_{p}}, \forall i \leq N P, \forall j \leq N R, \forall k \leq N_{\mathcal{S}_{j}} \\
& I_{p}=C T \text { ratio } \times \text { tap }, \forall j \leq N R, \forall k \leq N_{\mathcal{S}_{j}} \\
& I_{p} \geq I_{\text {pre-fault }}, \forall j \leq N R, \forall k \leq N_{\mathcal{S}_{j}} \\
& I c c / C T_{\text {prim }} \leq 20, \forall j \leq N R, \forall k \leq N_{\mathcal{S}_{j}} \\
& \underline{t a p} \leq t a p \leq \overline{t a p}, \forall j \leq N R, \forall k \leq N_{\mathcal{S}_{j}} \\
& \underline{T M S} \leq T M S \leq \overline{T M S}, \forall j \leq N R, \forall k \leq N_{\mathcal{S}_{j}} \\
& \alpha=\frac{n}{m} \\
& 0 \leq n \leq m \\
& 0 \leq \beta \leq 1 \\
& \mathcal{S}_{j} \ni \varsigma_{j k} \\
& \forall i, j, k, n, m \in \mathbb{N} \text {. }
\end{aligned}
$$

where: $t$ is the operating time ruled by the IEC 60255 standard (Equation 1f), being divided in $t^{\prime}$ - primary relay - and $t^{\prime \prime}$ - secondary relay (Equation 1a); NP is the number of scenarios (types and locations of the short-circuit); $N R$ is the number of the installed relays (primary); $\mathcal{S}_{j}$ is the set of secondary relays, such as 
$\left\{\varsigma_{j 1}, \varsigma_{j 2}, \varsigma_{j(\ldots)}, \varsigma_{j(k-1)}, \varsigma_{j k}\right\}$, of the primary relay $j$; $N_{\varsigma_{j}}$ is the number of elements in $\mathcal{S}_{j} ; \Phi$ is the curve code; $A_{\Phi}$ and $B_{\Phi}$ are coefficients dependent on $\Phi$; $\gamma$ is a multiplicative factor of $C T I ; C T I_{\max }$ is the maximum $C T I$ defined by the user; $\alpha$ and $n$ are the proportion and the absolute number of pairs of relays which may suffer decrease in $C T I$, respectively; $m$ is the total number of pairs of relays (number of constraints type Equation 1c); $I_{c c}$ is short-circuit current; beta is the percentage decrease of $C T I_{\max }$; relay tap is selected according to the input current $I_{\text {pre-fault }} ; C T_{\text {prim }}$ is the rated primary current of CT; $I_{\text {pre-fault }}$ is the pre-fault current; tap and $\underline{T M S}$ are lowest tap and TMS, respectively; $\overline{\text { tap }}$ and $\overline{T M S}$ are greatest tap and $T M S$, respectively.

The greater the coordination index goes, the greater the sum of operating time becomes, consequently being a contrasting condition. A low coordination index speeds up the operation of the DORs by decreasing the CTIs, however, high index lengthen the CTI if the involved devices require it (depending on the technology). CT's should not saturate when the short-circuit current reaches its maximum amplitude, the protective devices must be reliable enough to perceive the minimum short-circuit current and they should not operate for load current.

Equation $1 \mathrm{~d}-1 \mathrm{e}$ and $1 \mathrm{~h}-1 \mathrm{l}$ are not directly influenced by the type and location of short-circuit current during the simulation. In addition, $f_{2}$ only is unitary when all pairs have maximum $C T I$. Equation 1d means that the number of $\gamma$ 's equal to 1 should be $(1-\alpha) m$ at least and Equation $1 \mathrm{e}$ represents the percent range of $C T I$, being the parameter $\alpha$ inserted in the step function. When $\alpha$ is equal to zero, $\gamma$ will be automatically equal to one for all situations, constraining better the search space.

The problem model has a discrete non-linear nature. The proposal in this work is to transform the hard constraint which deals with CTI (fixed value) into a soft constraint, while still respecting the limits of the protection device. For example, a typical CTI for microprocessor based protective relays is between 0.1 and $0.2 \mathrm{~s}$, while for electromechanical devices is 0.3 to $0.4 \mathrm{~s}$ (Mansour et al., 2007). Therefore, if this range is satisfied in this case, our problem will be satisfied. The inspiration to design the method comes from (Chinneck, 2008), which alters some constraints to achieve feasibility in the context of a radiation therapy planning problem.

As can be seen, the mathematical formulation imposes conflicting specification on the technical performance of a given system arrangement. To examine the trade-offs between these conflicting objectives and to check the available options, an multiobjective algorithm that needs the designer's involvement as a decision maker has to be formu- lated. For reaching this goal, the multiobjective C-DEEPSO is proposed in Section 3. Following, the probabilistic distributions for all decision variables are in Section 4.

\section{An Overview About the Multiobjective C-DEEPSO}

C-DEEPSO is an advanced variant of the metaheuristic EPSO, which incorporates features of Evolutionary Computation, Particle Swarm Optimization (PSO) and Differential Evolution (DE) in a successful hybrid. These algorithms have been employed in their single objective version in many power system problems and industrial applications. For instance, in (Marcelino et al., 2016), it was adopted to enhance the reactive power of wind farms. C-DEEPSO uses a collective memory instead of numerous and independent memories which incorporates the search search experience of each particle

Considering the total of $n p$ particles in the simulation, the Movement Rule is given by Equation 2, Equation 3 and Equation 4. C-DEEPSO performs successive evaluations by using a population of solutions. Each individual $i$ travels the search space, reaching a position $X_{t-1}^{i}$ in each iteration $t-1$. The term velocity $V_{t}^{i}$ modifies this position to the next iteration $t . w_{I}^{i}, w_{A}^{i}$ and $w_{C}^{i}$ are the weights relating to inertia, assimilation and communication, respectively. The superscript * means that the corresponding variable suffers a mutation stage while the simulation occurs. $\Psi$ is essential to verify whether the particle $X_{r}$, based on its fitness value, will influence positively or not in that specific iteration.

$$
\begin{gathered}
V_{t}^{i}=w_{I}^{i, *} \times V_{t-1}^{i}+w_{A}^{i, *} \times\left(X_{b e s t}^{i}+F \times \Psi\right) \\
+w_{C}^{i, *} \times C_{t-1} \times\left(X_{g b}^{*}-X_{t-1}^{i}\right) \\
\Psi= \begin{cases}X_{r}-X_{t-1}^{i}, & \text { if } f\left(X_{r}\right) \leq f\left(X_{t-1}^{i}\right) \\
X_{t-1}^{i}-X_{r}, & \text { otherwise }\end{cases} \\
X_{t}^{i}=X_{t-1}^{i}+V_{t}^{i}
\end{gathered}
$$

Firstly, $X_{\text {best }}^{i}$ is the individual best position ever found and $X_{g b}$ is the best position ever found by the swarm (they are refined later to meet our goal). $F$ is a quantity which controls the amplification of differential variation, generally between zero and two. $C$ means a diagonal matrix with $n \times n$ dimension sampled at each iteration and respects a Bernoulli distribution which takes the value 1 (referring to the activated cooperation term) with probability $\rho$, being here named as communication probability rate. Secondly, $X_{r}$ is an individual that can be obtained based on one of the following hybrid strategy: $S_{g} P_{b}-r n d$, which guarantees a more appreciable assimilation of the search space(Marcelino et al., 2016). 
C-DEEPSO has a memory apparatus called Memory $B$ with size $\phi_{B}$ pre-defined by the user, being always less or equal to the total number of particles. By concatenating $B$ with all particles of the current generation and keeping their dimension $n$, a new matrix is formed to find completely all elements in $S_{g} P_{b}-r n d$ scheme. $X_{r}$ is randomly sampled from this matrix (uniform distribution). The random position $\nu$ must lie between 1 and $\left(\phi_{B}+n p\right)$ size. An effective way to save computational memory is just to observe whether $\nu \leq \phi_{B}$. Being affirmative, then $X_{r}=B^{\nu}$. Otherwise, $X_{r}=X_{t-1}^{\nu-\phi_{B}}$.

Moreover, the described weights are mutated according to Equation 5. The mutated weights must not be less than 0 and greater than 1. $\tau$ and $\tau^{\prime}$ are the mutation rate determined by the user. $N(0,1)$ is a random number from the standard normal distribution.

$$
w^{i, *}=w^{i}+\tau \times N(0,1)^{i}
$$

To avoid the swarm to be trapped in certain region, $X_{g b}$ also is moderately modified by applying normal distribution as described in Equation 6.

$$
X_{g b}^{i, *}=X_{g b}+\tau^{\prime} \times N(0,1)^{i}
$$

By using these basic definitions, the solutions with similar Pareto dominance rank (same front) are sorted in ascending order. For this, find initially all nondominated particles and assign rank $=1$. Ignore temporarily these elements to achieve the next ones. Continue this process until the entire swarm to be properly classified to obtain each front. Similarly to NSGA-II, we also generate offspring from ancestors, and apply a crowdedcomparison operator $\left(\prec_{c}\right)$ to elect each solution $a$ toward a uniformly spread front via crowding distance metric $\left(a_{\text {dist }}\right)$ and reject part of the solutions. The term $X_{g b}$ in Equation 2 is replaced with $X_{T \%}$. It is a position which is taken randomly from the top nondominated solutions ever found by the swarm (e.g. best $5 \%$ ). $X_{\text {best }}$ is modified only when the new particle is nondominated and it dominates the previous $X_{\text {best }}$. Finally, the Multiobjective C-DEEPSO can be summarized according to Algorithm 1.

\section{Probabilistic Distribution for Relay Coordination}

An important aspect to be discussed is the probabilistic distribution of the initial random values used for the relay coordination problem. In order to obtain quality solution in shortest time by using less evaluations, it is interesting that the most decision variables here, $T M S, I_{p}, \Phi$ and $\gamma$ are already initially located in a region with expected promisin solutions. It is also worth remembering



that the procedure does not prevent from visiting distinct and distant regions (assuming the expected region is known), but to a lesser extent.

It is known that probability distribution plays an important role to solve a number of real problems, such as to model the energy consumption of electric vehicles (normal), to estimate the average life cycle of equipment in electrical systems (exponential) and to represent circuit breaker closing times.

This paper examines four types of distribution: $a$. uniform; $b$. triangular; $c$. normal and; $d$. chi-squared one. The normal and chi-squared probability distribution need to be properly organized in order to comply the minimum and maximum limits of the protective devices. Each one is discussed in the following.

\subsection{Normal distribution}

Firstly, the standard normal distribution with zero median and unit variance should be denormalized, i.e. by transforming $[-Z \sigma ; Z \sigma]$, being $Z \approx 3$, into $\left[x_{i_{\text {min }}} ; x_{i_{\text {max }}}\right]$, being $x_{i}$ equal to $T D S_{i}$, $P S M_{i}$ or $\Phi_{i}$ of one given relay $i$ (Figure 1(a)). For matching the range according to the param- 
eter of the protective devices, Equation 7, Equation 8 and Equation 9 are used in the simulation. $Z$ is equals to the standard score obtained by the random number generator. The result of this correction may be viewed in Figure $1(\mathrm{~b}) . x$ equal to $T D S$ is the decision variable to be used in the optimization. After calculations, $\mu=1.6, \sigma=0.4406$ and the denominator of Equation 8 is equal to 3.6314 , in this case.

$$
\begin{gathered}
\mu_{i}=\frac{x_{i_{\max }}-x_{i_{\min }}}{2}+x_{i_{\min }} \\
\sigma_{i}=\frac{x_{i_{\max }}-\mu_{i}}{\max \left\{\max \left(Z^{+}\right) ;-\min \left(Z^{-}\right)\right\}} \\
x_{i}=Z \sigma_{i}+\mu_{i}
\end{gathered}
$$

Figure 1: Frequency and PDF of the normal distribution

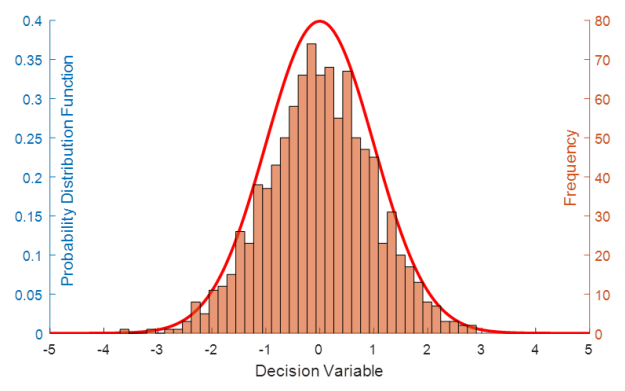

(a) $Z$ is the decision variable, $\mu=0$ and $\sigma=1$.



(b) Employing the denormalization.

\subsection{Chi-squared distribution}

The chi-squared distribution leads to the same reasoning, where the original values should also be rescaled to fullfil the minimum and maximum values of the relay settings. For this, use the mathematical formulation given in Equation 10 and Equation 11 to transform linearly $\left[l_{i_{\text {min }}}<l_{i}<\right.$ $\left.l_{i_{\max }}\right]$ into $\left[x_{i_{\min }}<x_{i}<x_{i_{\max }}\right]$ for each DOR $i$.

$$
\begin{gathered}
\gamma_{i}=\frac{l_{i}-l_{i_{\text {min }}}}{l_{i_{\text {max }}}-l_{i_{\text {min }}}} \\
x_{i}=\gamma_{i} \times\left(x_{i_{\text {max }}}-x_{i_{\text {min }}}\right)+x_{i_{\text {min }}}
\end{gathered}
$$

Figure 2 shows the chi-squared distribution complying the inferior and superior bounds of the variable $T D S$. Degrees of freedom are $v=2$ and noncentrality parameter is $\delta=0$ in Figure 2(a). In Figure 2(b), $\left[x_{i_{\text {min }}} ; x_{\text {imax }}\right]=$ $\left[T D S_{i_{\text {min }}} ; T D S_{\text {imax }}\right]=[0 ; 3.2]$.
Figure 2: Frequency and PDF of the chi-squared distribution



(a) $l$ is the decision variable, being approximately equal to 14 .



(b) Finding $x$ using linear transformation.

\subsection{Uniform and triangular distributions}

The uniform and triangular distribution do not need any scale transformation, because the minimum and maximum limits are inserted directly and this procedure normally is well performed in programming languages such as $\mathrm{C}++$ or Matlab ${ }^{\circledR}$ (The MathWorks).

\section{Results}

The results of the Multiobjective C-DEEPSO will be discussed in this part. As stopping criteria, the simulation stops after a specified number of iterations or consecutive iterations without significant changes in the solutions, adopting a tolerance of $10^{-3}$ between the previous and current iterations.

The IEEE 30-bus test system (Figure 3) is used in this investigation. It represents two subsystems (132-kV subtransmission system and 33-kV distribution system), a well-known standard benchmark network of the American Electric Power system. We consider different shortcircuits, fault locations (close-in, intermediate point and remote bus) and scenarios that represent contingency states. The link between them is composed by four step-down transformers. Each line is equipped with a circuit breaker as well as a DOR with inverse characteristics, with $P S M$ and $T D S$ in the range of $0.25-20\left(I_{p}\right.$ is directly obtained by using Equation $1 \mathrm{~g}$ and Equation $1 \mathrm{~h}$ ) and $0.05-3.2$, respectively. Finally, a CTI varying of $200 \mathrm{~ms}$ down to a percentage decrease of $40 \%$ has been adopted for all cases.

Figure 4 shows four Pareto curves in rela- 
Figure 3: IEEE 30-bus test system.



tion to the number of particles after 100 iterations. Note that 100 particles (red color) lead to the algorithm to converge faster, minimizing $f_{1}$ with the set of inequality constraints replaced by a penalty function as well as maximizing $f_{2}$. In addition, observe that the case with 75 particles (green color) has a better spreading of solutions (see its extremes $=\left\{2.42 \times 10^{11} ;-0.902314\right\}$ and $\left.\left\{2.75 \times 10^{14} ;-1\right\}\right)$. It is important to know the trade-off among the curves and these characteristics. $t$ equal to $10^{3}$ has been adopted in the simulation when the current seen by the relay is lower than $I_{p}$. The cardinalities among the cases are respectively, 10, 18, 9 and 10 .

Based on the solutions extracted from Figure 4, Table 1 shows the values of decision variables using 100 particles: the first 20 relays and the first 38 pairs of primary and backup $(\mathrm{P} / \mathrm{B})$ relays. Note that solution 11 has all $\gamma$ equal to 1 , while the inverse situation occurs in Solution 1. In addition, $\Phi$ for each relay can be equal to 1 (Inverse), 2 (Very Inverse) or 3 (Extremely Inverse). Applying a close-in short-circuit, the number of
Figure 4: Pareto fronts versus number of particles.

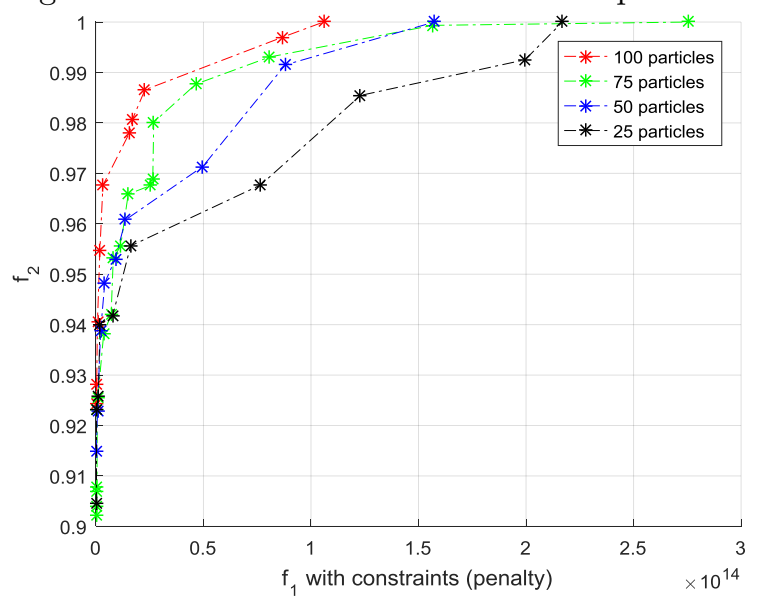

non-satisfied pairs were 19, 22, 10, 6 and 5 out of 175 constraints, respectively. On the other hand, when the short-circuit was applied at the remote bus, the values were $29,25,18,15$ and 16 out of 175. See that greater $\gamma$ lead to less miscoordination, but the operating time is negatively affected.

Furthermore, it is notorious that the performance of the algorithm is strongly influenced by the choice where the initial random points are positioned. Table 2 shows the final results of different probability distributions in relation to the decision variables $I p, T M S$ and curve type. As can be seen, six different tests were carried out by changing the type of distribution. The criteria stopping adopted was the cardinality value without change for 20 successive iterations. This value is shown in column $F C$. Observe that the convergence speed when using triangular and chi-square distributions in $T M S$ or $I_{p}$ are considerably better than using uniform distribution, but the indicator $F C$ is lower, indicating few solutions. The absolute difference between the maximum and minimum times is $212.34 \mathrm{~s}$. Therefore, a reduction of $57.10 \%$ in this parameter.

This technique is very useful to investigate and fullfill two aspects for the protection system reliability: dependability and security. Dependability-related failure occurs when a device does not operate properly at the required time. In this case, the backup DOR can have shorter operating time, acting before its primary one. A security-related failure occurs when the relay operates faster than designed (inappropriate opening of a circuit). In the simulation, several levels of operating time and coordination are shown for one option to be chosen according to the phylosophy of protective relaying.

The algorithm has been executed under ISO $\mathrm{C}++2011$ standard to design the code by handling Eclipse free-software (version Luna 4.4.2). The MinGW compiler, which includes GCC compilers, and Boost source libraries (version 1.61.0) has been used. The processor is an Intel i3 (2.13 $\mathrm{GHz})$ and 8 GB RAM running MS Windows OS, 
Table 1: Decision variables of Pareto front (100 particles).

\begin{tabular}{|c|c|c|c|c|c|c|c|c|c|c|c|}
\hline Variables & S1 & $\mathrm{S} 2$ & S3 & S10 & S11 & Variables & S1 & S2 & S3 & S10 & S11 \\
\hline$T M S_{1}$ & 0.364 & 0.8 & 0.125 & 0.8 & 0.151 & $\Phi_{10}$ & 2 & 3 & 3 & 3 & 3 \\
\hline$T M S_{2}$ & 0.222 & 0.8 & 0.8 & 0.263 & 0.374 & $\Phi_{11}$ & 2 & 2 & 3 & 3 & 3 \\
\hline$T M S_{3}$ & 0.777 & 0.142 & 0.8 & 0.8 & 0.8 & $\Phi_{12}$ & 2 & 1 & 2 & 3 & 3 \\
\hline$T M S_{4}$ & 0.607 & 0.641 & 0.8 & 0.359 & 0.491 & $\Phi_{13}$ & 2 & 1 & 3 & 3 & 3 \\
\hline$T M S_{5}$ & 0.664 & 0.8 & 0.8 & 0.8 & 0.8 & $\Phi_{14}$ & 2 & 1 & 3 & 3 & 3 \\
\hline$T M S_{6}$ & 0.672 & 0.74 & 0.05 & 0.8 & 0.8 & $\Phi_{15}$ & 2 & 3 & 3 & 3 & 3 \\
\hline$T M S_{7}$ & 0.8 & 0.67 & 0.8 & 0.8 & 0.8 & $\Phi_{16}$ & 2 & 1 & 3 & 3 & 3 \\
\hline$T M S_{8}$ & 0.261 & 0.626 & 0.8 & 0.8 & 0.8 & $\Phi_{17}$ & 2 & 1 & 3 & 3 & 3 \\
\hline$T M S_{9}$ & 0.743 & 0.417 & 0.8 & 0.8 & 0.8 & $\Phi_{18}$ & 2 & 2 & 3 & 3 & 3 \\
\hline$T M S_{10}$ & 0.164 & 0.8 & 0.8 & 0.426 & 0.35 & $\Phi_{19}$ & 2 & 3 & 3 & 3 & 3 \\
\hline$T M S_{11}$ & 0.29 & 0.679 & 0.8 & 0.8 & 0.535 & $\Phi_{20}$ & 2 & 3 & 3 & 3 & 3 \\
\hline$T M S_{12}$ & 0.403 & 0.8 & 0.8 & 0.8 & 0.8 & $\gamma_{1}$ & 0.871 & 0.928 & 1 & 1 & 1 \\
\hline$T M S_{13}$ & 0.133 & 0.355 & 0.655 & 0.8 & 0.05 & $\gamma_{2}$ & 0.919 & 1 & 1 & 1 & 1 \\
\hline$T M S_{14}$ & 0.393 & 0.244 & 0.8 & 0.8 & 0.8 & $\gamma_{3}$ & 0.987 & 1 & 1 & 1 & 1 \\
\hline$T M S_{15}$ & 0.709 & 0.8 & 0.8 & 0.8 & 0.8 & $\gamma_{4}$ & 0.987 & 0.864 & 0.944 & 1 & 1 \\
\hline$T M S_{16}$ & 0.378 & 0.435 & 0.799 & 0.8 & 0.8 & $\gamma_{5}$ & 1 & 0.912 & 0.992 & 1 & 1 \\
\hline$T M S_{17}$ & 0.639 & 0.654 & 0.8 & 0.8 & 0.8 & $\gamma_{6}$ & 0.882 & 0.983 & 1 & 1 & 1 \\
\hline$T M S_{18}$ & 0.33 & 0.297 & 0.8 & 0.8 & 0.8 & $\gamma_{7}$ & 0.948 & 0.850 & 0.930 & 1 & 1 \\
\hline$T M S_{19}$ & 0.35 & 0.8 & 0.8 & 0.125 & 0.491 & $\gamma_{8}$ & 0.935 & 1 & 1 & 1 & 1 \\
\hline$T M S_{20}$ & 0.8 & 0.8 & 0.8 & 0.8 & 0.8 & $\gamma_{9}$ & 0.929 & 0.9 & 0.98 & 1 & 1 \\
\hline$I_{p_{1}}$ & 1318.65 & 1489.6 & 2029.12 & 2073.6 & 2073.6 & $\gamma_{10}$ & 1 & 0.834 & 0.914 & 1 & 1 \\
\hline$I_{p_{2}}$ & 1375.82 & 1101.56 & 1641.08 & 2073.6 & 2073.6 & $\gamma_{11}$ & 1 & 0.973 & 1 & 1 & 1 \\
\hline$I_{p_{3}}$ & 1143.54 & 1934.4 & 1934.4 & 1934.4 & 1934.4 & $\gamma_{12}$ & 1 & 0.945 & 1 & 1 & 1 \\
\hline$I_{p_{4}}$ & 1163.43 & 1047.58 & 1352 & 1352 & 1352 & $\gamma_{13}$ & 0.829 & 0.958 & 1 & 0.989 & 1 \\
\hline$I_{p_{5}}$ & 791.841 & 745.175 & 1119.17 & 1352 & 1352 & $\gamma_{14}$ & 0.981 & 0.872 & 0.952 & 1 & 1 \\
\hline$I_{p_{6}}$ & 627.23 & 859.169 & 1232.77 & 1374.43 & 1413 & $\gamma_{15}$ & 0.989 & 0.824 & 0.904 & 1 & 1 \\
\hline$I_{p_{7}}$ & 809.257 & 633.233 & 985.633 & 1357 & 1357 & $\gamma_{16}$ & 0.91 & 0.873 & 0.953 & 1 & 1 \\
\hline$I_{p_{8}}$ & 1051.93 & 729.837 & 1147.44 & 1526 & 1526 & $\gamma_{17}$ & 0.988 & 0.969 & 1 & 1 & 1 \\
\hline$I_{p_{9}}$ & 769.269 & 325.599 & 616.399 & 886 & 886 & $\gamma_{18}$ & 1 & 0.844 & 0.924 & 1 & 1 \\
\hline$I p_{10}$ & 946.024 & 548.553 & 1077.75 & 1494 & 1494 & $\gamma_{19}$ & 0.977 & 0.874 & 0.954 & 1 & 1 \\
\hline$I_{p_{11}}$ & 1283.42 & 567.604 & 1028.4 & 1291 & 1291 & $\gamma_{20}$ & 0.918 & 0.877 & 0.957 & 1 & 1 \\
\hline$I_{p_{12}}$ & 973 & 973 & 973 & 973 & 973 & $\gamma_{21}$ & 0.954 & 1 & 1 & 1 & 1 \\
\hline$I_{p_{13}}$ & 1594.09 & 908.295 & 1579.5 & 2000 & 2000 & $\gamma_{22}$ & 0.827 & 1 & 1 & 0.987 & 1 \\
\hline$I_{p_{14}}$ & 864.182 & 780.089 & 964 & 964 & 964 & $\gamma_{23}$ & 0.826 & 0.879 & 0.959 & 0.986 & 1 \\
\hline$I_{p_{15}}$ & 691.182 & $\begin{array}{l}1296.02 \\
\end{array}$ & 1992.42 & 2000 & 2000 & $\gamma_{24}$ & 0.963 & 0.904 & 0.984 & 1 & 1 \\
\hline$I_{p_{16}}$ & 1330.63 & 800.646 & 1477.45 & 2000 & 2000 & $\gamma_{25}$ & 0.955 & 0.979 & 1 & 1 & 1 \\
\hline$I_{p_{17}}$ & 1274.46 & 1303.14 & 1959.14 & 2000 & 2000 & $\gamma_{26}$ & 0.917 & 0.822 & 0.902 & 1 & 1 \\
\hline$I_{p_{18}}$ & 641.575 & 497.623 & 893.623 & 1254 & 1254 & $\gamma_{27}$ & 0.929 & 0.99 & 1 & 1 & 1 \\
\hline$I_{p} p_{19}$ & 709.239 & 768.258 & 862 & 862.000 & 862 & $\gamma_{28}$ & 0.913 & 0.875 & 0.955 & 1 & 1 \\
\hline$I_{p_{20}}$ & 399.46 & 459.192 & 515 & 515 & 515 & $\gamma_{29}$ & 0.967 & 0.948 & 1 & 1 & 1 \\
\hline$\Phi_{1}$ & 2 & 3 & 3 & 3 & 3 & $\gamma_{30}$ & 0.854 & 0.833 & 0.913 & 1 & 1 \\
\hline$\Phi_{2}$ & 2 & 3 & 3 & 3 & 3 & $\gamma_{31}$ & 1 & 0.861 & 0.941 & 1 & 1 \\
\hline$\Phi_{3}$ & 2 & 3 & 3 & 3 & 3 & $\gamma_{32}$ & 0.864 & 0.17 & 0.997 & 1 & 1 \\
\hline$\Phi_{4}$ & 2 & 2 & 3 & 3 & 3 & $\gamma_{33}$ & 0.847 & 0.73 & 1 & 1 & 1 \\
\hline$\Phi_{5}$ & 2 & 3 & 3 & 3 & 3 & $\gamma_{34}$ & 0.43 & 0.975 & 1 & 1 & 1 \\
\hline$\Phi_{6}$ & 2 & 2 & 3 & 3 & 3 & $\gamma_{35}$ & 0.48 & 0.982 & 1 & 1 & 1 \\
\hline$\Phi_{7}$ & 2 & 3 & 3 & 3 & 3 & $\gamma_{36}$ & 0.78 & 0.958 & 1 & 1 & 1 \\
\hline$\Phi_{8}$ & 2 & 3 & 3 & 3 & 3 & $\gamma_{37}$ & 0.12 & 0.839 & 0.919 & 1 & 1 \\
\hline$\Phi_{9}$ & 2 & 3 & 3 & 3 & 3 & $\gamma_{38}$ & 0.3 & 0.894 & 0.974 & 0.99 & 1 \\
\hline
\end{tabular}

Table 2: Influence of the probability distribution on optimization performance.

\begin{tabular}{|c|c|c|c|c|c|}
\hline Tests & Variables & DIST & IT & Time [s] & $F C$ \\
\hline \multirow[b]{2}{*}{1} & $T M S$ & Uniform & \multirow[b]{2}{*}{258} & \multirow[b]{2}{*}{371.86} & \multirow[b]{2}{*}{8} \\
\hline & $\begin{array}{c}I_{p} \\
\Phi \text { and } \gamma\end{array}$ & $\begin{array}{l}\text { Uniform } \\
\text { Triangular }\end{array}$ & & & \\
\hline \multirow[b]{2}{*}{2} & $T M S$ & Uniform & \multirow[b]{2}{*}{209} & \multirow[b]{2}{*}{351.96} & \multirow[b]{2}{*}{11} \\
\hline & $I_{p}$ & Uniform & & & \\
\hline \multirow[b]{2}{*}{3} & $T M S$ & Triangular & \multirow[b]{2}{*}{81} & \multirow[b]{2}{*}{151.81} & \multirow[b]{2}{*}{9} \\
\hline & $\begin{array}{c}I_{p} \\
\Phi \text { and } \gamma\end{array}$ & $\begin{array}{c}\text { Triangular } \\
\text { Normal }\end{array}$ & & & \\
\hline \multirow[b]{2}{*}{4} & $T M S$ & Triangular & \multirow[b]{2}{*}{126} & \multirow[b]{2}{*}{201.30} & \multirow[b]{2}{*}{9} \\
\hline & $\begin{array}{c}I_{p} \\
\Phi \text { and } \gamma\end{array}$ & $\begin{array}{l}\text { Triangular } \\
\text { Uniform }\end{array}$ & & & \\
\hline \multirow[b]{2}{*}{5} & $T M S$ & Chi-Squared & \multirow[b]{2}{*}{116} & \multirow[b]{2}{*}{186.87} & \multirow[b]{2}{*}{5} \\
\hline & $\begin{array}{c}I_{p} \\
\Phi \text { and } \gamma\end{array}$ & $\begin{array}{c}\text { Chi-Squared } \\
\text { Normal }\end{array}$ & & & \\
\hline \multirow[b]{2}{*}{6} & $T M S$ & Chi-Squared & \multirow[b]{2}{*}{79} & \multirow[b]{2}{*}{159.52} & \multirow[b]{2}{*}{6} \\
\hline & $\begin{array}{c}I_{p} \\
\Phi \text { and } \gamma\end{array}$ & $\begin{array}{c}\text { Chi-Squared } \\
\text { Triangular }\end{array}$ & & & \\
\hline
\end{tabular}

64 bits.

\section{Conclusion}

This paper reports the use of a multiobjective technique developed from the original C-DEEPSO to coordinate efficiently DORs. The strategy has been devised by solving two objective functions which treat the total operating time of these DORs. The coordination index allows obtaining a greater set of solutions which gives better options to the protection engineer. This index when unitary (best situation for $f_{2}$ ) guarantees the indicated maximum CTI is found, which coordination between the main and backup relays are plenty coordinated, however it is expected to have greater operating time ( $f_{1}$ gets worst performance). On the other hand, when this index is decreased, respecting the margin established by the expert, $f_{1}$ may get a better efficiency.

This trade-off has corroborated the use of a multiobjective approach to study the coordination without needing to modify the number of constraints (relay pairs) commonly used for DOR coordination. A set of Pareto-optimal or non-dominated solutions do not exclude a solution without inserting some specification defined by the user preference. C-DEEPSO represents a capable step towards solving other problems in power systems involving two or even more objectives.

A hybrid Particle Swarm Optimization algorithm has been engineered in this paper, being that performance comparison, with a naive approach or other possible algorithms, can be provided in further investigation.

Furthermore, depending on the number of particles, the algorithm has shown different performances in terms of algorithm convergence and the diversity of the solution. Better Pareto front has been obtained when using 100 particles, in- 
stead of 25 particles. Another point discussed in the work has been about the probabilistic distribution to initialize the solutions, which when used adequately can enhance the solution significantly. We suggested to use triangular and chi-squared distribution to coordinate DORs in optimization tools.

\section{Agradecimentos}

This scientific work has been supported by the São Paulo Research Foundation - FAPESP (grants n. 2012/25292-1|link and n. 2014/27342-1|link) and the European Commission's ERASMUS programme (BE MUNDUS Project) under application n. BM15DM0973 (2016/2017).

\section{References}

Bernardes, W. M. S., Asada, E. N. and d. M. Vieira, J. C. (2015). Topological processing of mutually coupled circuits for directional overcurrent protection, 2015 IEEE Power Energy Society General Meeting, pp. 1-5.

Bernardes, W. M. S., Santos, F. M. P., Asada, E. N., Souza, S. A. and Ramos, M. J. (2013). Application of discrete PSO and evolutionary PSO to the coordination of directional overcurrent relays, 2013 IEEE XVII International Conference on Intelligent System Applications to Power Systems (IEEE ISAP 2013), pp. 1-6.

Bottura, F. B., Bernardes, W. M. S., Oleskovicz, M. and Asada, E. N. (2017). Setting directional overcurrent protection parameters using hybrid GA optimizer, Elec. Power Syst. Research 143: 400 - 408.

Chinneck, J. W. (2008). Feasibility and Infeasibility in Optimization: Algorithms and Computational Methods, 1st edn, Springer US.

Costa, M. H., Saldanha, R. R., Ravetti, M. G. and Carrano, E. G. (2017). Robust coordination of directional overcurrent relays using a matheuristic algorithm, IET Generation, Transmission Distribution 11(2): 464-474.

El-Fergany, A. A. and Hasanien, H. M. (2017). Optimized settings of directional overcurrent relays in meshed power networks using stochastic fractal search algorithm, Int. Trans. on Elec. Energy Syst. pp. e2395-n/a.

Elmitwally, A., Gouda, E. and Eladawy, S. (2015). Optimal allocation of fault current limiters for sustaining overcurrent relays coordination in a power system with distributed generation, Alexandria Engineering Journal 54(4): 1077 - 1089 .
IEEE (2001). IEEE recommended practice for protection and coordination of industrial and commercial power systems, IEEE Std 2422001 (Revision of IEEE Std 242-1986) [IEEE Buff Book] pp. 1-710.

Mansour, M. M., Mekhamer, S. F. and ElKharbawe, N. (2007). A modified particle swarm optimizer for the coordination of directional overcurrent relays, IEEE Transactions on Power Delivery 22(3): 1400-1410.

Marcelino, C. G., Almeida, P. E. M., Wanner, E. F., Carvalho, L. M. and Miranda, V. (2016). Fundamentals of the C-DEEPSO algorithm and its application to the reactive power optimization of wind farms, IEEE Congress on Evolut. Computation (CEC), pp. 1547-1554.

Meskin, M., Domijan, A. and Grinberg, I. (2015). Optimal co-ordination of overcurrent relays in the interconnected power systems using break points, Electric Power Systems Research 127: $53-63$.

Miranda, V. and Fonseca, N. (2002). Epso best-of-two-worlds meta-heuristic applied to power system problems, Proceedings of the 2002 Congress on Evolutionary Computation. CEC'02 (Cat. No.02TH8600), Vol. 2, pp. 1080-1085 vol.2.

Noghabi, A. S., Mashhadi, H. R. and Sadeh, J. (2010). Optimal coordination of directional overcurrent relays considering different network topologies using interval linear programming, IEEE Transactions on Power Delivery 25(3): 1348-1354.

Rajput, V. N., Adelnia, F. and Pandya, K. S. (2018). Optimal coordination of directional overcurrent relays using improved mathematical formulation, IET Generation, Transmission and Distribution 12: 2086-2094(8).

Shabani, M. and Karimi, A. (2018). A robust approach for coordination of directional overcurrent relays in active radial and meshed distribution networks considering uncertainties, International Transactions on Electrical Energy Systems 28(5): e2532.

Tjahjono, A., Anggriawan, D. O., Faizin, A. K., Priyadi, A., Pujiantara, M., Taufik, T. and Purnomo, M. H. (2017). Adaptive modified firefly algorithm for optimal coordination of overcurrent relays, IET Generation, Transmission Distribution 11(10): 2575-2585.

Ukil, A., Deck, B. and Shah, V. H. (2011). Current-only directional overcurrent relay, IEEE Sensors Journal 11(6): 1403-1404. 